\title{
Of blood and bone: the sotatercept adventure
}

Severe persistent anaemia remains the most common problem that haematologists are facing when treating patients with myelodysplastic syndromes. ${ }^{1} \quad \mathrm{New}$ effective and safe drugs with an easy and simple method of administration are urgently needed for outpatients, to allow long-term treatment that improves their quality of life. Patients with lower-risk myelodysplastic syndromes generally live much longer than patients with higher-risk disease and thus, often have many consecutive treatment attempts to avoid red blood cell transfusions. ${ }^{1}$ The study by Rami Komrokji and colleagues $^{2}$ published in The Lancet Haematology efficiently contributes to the identification of a therapy that can relieve anaemia in patients with lower-risk myelodysplastic syndromes who have had previous treatments. In this phase 2 dose-ranging study, sotatercept was administered to 74 patients with lower-risk myelodysplastic syndromes in whom previous treatment had failed and who were transfusion dependent. Patients had either primary resistance or had lost response to therapies. Overall erythroid haematological improvement (HI-E) was achieved by 36 (49\%; 95\% Cl 38-60) of the 74 patients treated with sotatercept of all doses; with $21(60 \% ; 44-75)$ of 35 patients treated with $1 \mathrm{mg} / \mathrm{kg}$ achieving HI-E. Notably, very few patients had cytopenias other than anaemia, but approximately half of the patients with low platelet counts or neutrophil counts achieved platelet or neutrophil haematological improvements.

Sotatercept, previously known as ACE-011, is a hybrid molecule: a fusion protein consisting of the extracellular domain of the human activin receptor type IIA and the crystallisable domain of the human immunoglobulin G1 (IgG1) fragment, and is administered by subcutaneous injection. The erythroidstimulating activity of sotatercept was discovered in post-menopausal women treated for osteoporosis with sotatercept ${ }^{3}$ because of its role in the modulation of bone resorption and formation. In fact, sotatercept competes extracellularly with the ligands of the TGF $\beta$ superfamily, activins, and bone morphogenic proteins, trapping them and blocking signal transduction via the SMAD signalling pathway. ${ }^{4}$ This inhibition of the TGF $\beta$ pathway, besides bone anabolic effects, relieves the aberrant blockage of the transcription of genes devoted to stimulation of late-stage erythropoiesis characteristic of some subtypes of myelodysplastic syndromes, including subtypes with ring sideroblasts with their specific pathogenetic molecular feature. ${ }^{5}$

The most intriguing aspect of sotatercept is indeed its mechanism of action. ${ }^{4}$ The evidence of erythroidstimulating and antiosteoporotic activity of this drug should prompt further study of the mutifaceted and extremely complex TGF $\beta$ pathway. ${ }^{6}$ In fact, the TGF $\beta$ pathway modulates different cellular functions in a cell type-specific and context-dependent manner thanks to a very fine tuning and interplay of receptors, co-receptors, and ligands. ${ }^{6}$ The complexity of the TGF $\beta$ pathway might be the reason why luspatercept (fusion of activinRIIB and IgG1), which has a very similar structure to sotatercept, does not show a completely overlapping spectrum of activity with sotatercept. ${ }^{\text {? }}$ Additionally, other TGF $\beta$ pathway inhibitors such as galunisertib, which acts by directly binding to the same family of receptors, do not seem to achieve such strong clinical effects on erythropoieisis as do sotatercept and luspatercept. ${ }^{8}$ These complex mechanisms of action must be better understood in order to optimise therapy for myelodysplastic syndromes, and most probably other illnesses too. ${ }^{9}$

What are the clinical characteristics of the patients with lower-risk myelodysplastic syndromes who are sensitive to sotatercept? Are these in a different category to those who are responsive to luspatercept, and what are the differences in their molecular characteristics? Although these questions remain unanswered, some important results have been generated by the study of Komrokji and colleagues. ${ }^{2}$ For example, the HI-E response to sotatercept was sustained for at least 56 days without transfusions by seven $(58 \%)$ of 12 patients with low transfusion burdens. On the basis of these results, although the sample of patients with low transfusion burdens was small, treatment with sotatercept should be started as soon as possible after erythropoiesisstimulating agent (ESA) failure. Regardless, responses were still achieved in patients who had received other drugs such as hypomethylating agents (21 [58\%] of 36 patients) or lenalidomide (19 [54\%] of 35 patients).

A large proportion of the patients included in the study had a diagnosis of refractory anaemia with ring
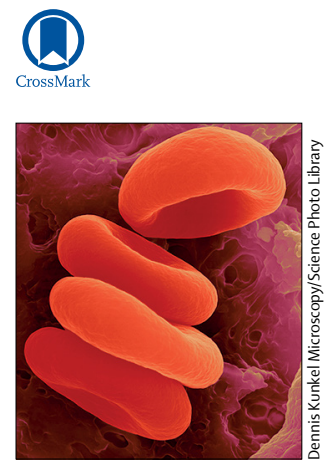

Published Online January 10, 2018 http://dx.doi.org/10.1016/ S2352-3026(18)30003-6 See Articles page e63 
sideroblasts ( $\geq 15 \%$ ring sideroblasts; 51 [69\%] of 74 patients), and a higher proportion of these patients achieved HI-E (30 [59\%] of 51) than that of patients without ring sideroblasts (four [22\%] of 18 patients). Patients belonging to the subgroup of ring sideroblastpositive myelodysplastic syndromes are abundant in all clinical studies of ESA-resistant lower-risk myelodysplastic syndromes, ${ }^{9,10}$ which might be because of their longer overall survival and early loss of response to ESAs. This fact could potentially bias analyses, and only randomised studies with larger cohorts of patients with myelodysplastic syndromes with and without ringed sideroblasts could clarify the issue.

In the study by Komrokji and colleagues, ${ }^{2}$ sotatercept showed a high erythroid-stimulating activity, but transfusion independence of at least 56 days was reached only in a minority of cases $(20$ [27\%, $95 \% \mathrm{Cl} 18-38]$ of 74 patients). This result is similar to those observed for other rescue treatments for patients with relapsed or refractory myelodysplastic syndromes, such as lenalidomide. $^{10}$ The study ${ }^{2}$ also showed a higher proportion of patients with responses to sotatercept in a subgroup who had lower concentrations of endogenous erythropoietin, although this difference was not significant. However, this confirms what has been shown in previous studies. ${ }^{710}$ Furthermore, treatmentrelated grade 3-4 adverse events were scarce, reported in four $(5 \%)$ of the 74 patients.

In conclusion, sotatercept is a promising and safe new approach to treat anaemia in patients with lowerrisk myelodysplastic syndromes. Ameliorating anaemia in myelodysplastic syndromes by reversing ineffective erythropoiesis secondary to aberrant TGF $\beta$ stimulation is indeed an interesting new therapeutic avenue for these patients. In order to optimise this approach, a more detailed picture of the downstream effects of sotatercept and of the drugs with similar molecular targets are needed, and the molecular and functional characteristics of the dysplastic diseases that can be responsive to such treatments need to be clearly defined.

\section{Valeria Santini}

MDS Unit, SOD Hematology, AOU Careggi, University of Florence, Florence 50134, Italy

valeria.santini@unifi.it

I gave lectures in supported symposia for Celgene, Janssen, and Novartis. I was on advisory boards for Abbvie, Otsuka, and Janssen.

1 Santini V. First-line therapeutic strategies for myelodysplastic syndromes. Clin Lymphoma Myeloma Leuk 2017; 17S: S31-36

2 Komrokji R, Garcia-Manero G, Ades L, et al. Sotatercept with long-term extension for the treatment of anaemia in patients with lower-risk myelodysplastic syndromes: a phase 2 dose-ranging trial. Lancet Haematol 2018; published online Jan 10. http://dx.doi.org/10.1016/S23523026(18)30002-4.

3 Ruckle J, Jacobs M, Kramer W, et al. Single-dose, randomized, double-blind placebo-controlled study of ACE-011 (ActRIIA-lgG1) in postmenopausal women. J Bone Miner Res 2009; 24: 744-52.

4 Fajardo RJ, Manoharan RK, Pearsall RS, et al. Treatment with a soluble receptor for activin improves bone mass and structure in the axial and appendicular skeleton of female cynomolgus macaques (Macaca fascicularis). Bone 2010; 46: 64-71.

5 Mies A, Hermine O, Platzbecker U. Activin receptor II ligand traps and their therapeutic potential in myelodysplastic syndromes with ring sideroblasts. Curr Hematol Malig Rep 2016; 11: 416-24.

6 Budi EH, Duan D, Derynck R. Transforming growth factor- $\beta$ receptors and smads: regulatory complexity and functional versatility. Trends Cell Biol 2017; 27: 658-72

7 Platzbecker U, Germing U, Götze KS, et al. Luspatercept for the treatment of anaemia in patients with lower-risk myelodysplastic syndromes (PACE-MDS): a multicentre, open-label phase 2 dose-finding study with long-term extension study. Lancet Oncol 2017; 18: 1338-47.

8 Valcarcel D, Verma A, Platzbecker U, et al. Phase 2 study of monotherapy galunisertib (LY2157299 monohydrate) in very low-, low-, and intermediate-risk patients with myelodysplastic syndromes. Blood 2015; 126: 1669.

9 Fields SZ, Parshad S, Anne M, et al. Activin receptor antagonists for cancerrelated anemia and bone disease. Expert Opin Investig Drugs 2013 22: 87-101

10 Santini V, Almeida A, Giagounidis A, et al. Randomized phase III study of lenalidomide versus placebo in RBC transfusion-dependent patients with lower-risk non-del(5q) myelodysplastic syndromes and ineligible for or refractory to erythropoiesis-stimulating agents. J Clin Oncol 2016; 34: $2988-96$ 